This document must be cited according to its final version which is published in a journal as:

P. Dufour ${ }^{1}$, S. Flila ${ }^{1}$, H. Hammouri ${ }^{1}$, "Observer design for MIMO non-uniformly observable systems", IEEE Transactions on Automatic Control, ISSN: 0018-9286 57(2), pp. 511-516, 2012. http://dx.doi.org/10.1109/TAC.2011.2166667

All open archive documents of Pascal Dufour are available at: http://hal.archives-ouvertes.fr/DUFOUR-PASCAL-C-3926-2008

The professional web page ( $\mathrm{Fr} / \mathrm{En}$ ) of Pascal Dufour is: http://www.lagep.univ-lyon1.fr/signatures/dufour.pascal

The professional web page ( $\mathrm{Fr} / \mathrm{En}$ ) of Hassan Hammouri is: http://www.lagep.univ-lyon1.fr/signatures/hammouri.hassan

The web page of this research group is:

http://hal.archives-ouvertes.fr/SNLEP

1

Université de Lyon, Lyon, F-69003, France; Université Lyon 1;

CNRS UMR 5007 LAGEP (Laboratoire d'Automatique et de GEnie des Procédés),

43 bd du 11 novembre, 69100 Villeurbanne, France

Tel +33 (0) 472431845 - Fax +33 (0) 472431699

http://www-lagep.univ-lyon1.fr/ http://www.univ-lyon1.fr http://www.cnrs.fr 


\section{Observer design for MIMO non-uniformly observable systems}

Pascal Dufour, Saida Flila and Hassan Hammouri

\begin{abstract}
The design of high gain observers is usually based on normal forms of observability. If the system is observable for every input (uniform observability), the gain of the observer does not required a solution to differential equation. For multiple input multiple output (MIMO) non-uniformly observable systems, we give here a sufficient condition that the input must satisfy in order to design an observer. Unlike uniformly observable systems, the observer gain of non-uniformly observable systems is derived from a Lyapunov differential equation.
\end{abstract}

Index Terms-Nonlinear systems, observers.

\section{INTRODUCTION}

The problem of state estimation is an important issue in control, diagnosis and monitoring of process systems. Many methods have been developed for designing an observer for nonlinear systems. Among these methods, a rather natural approach consists in steering the nonlinear system into a state affine system up to output injection, by a suitable change of coordinates. Indeed, an extended Luenberger (or Kalman) observer can be designed for this class of systems (see for instance [3], [4], [6], [5], [12], [7], [16], [18], [15], [22], [27]). From the observability point of view, nonlinear systems which can be steered into linear systems up to output injection by a change of coordinates ([3], [4], [6]) are similar to stationary linear systems, in the sense that their observability does not depend on the input and a Luenberger observer can be designed for both classes of systems. An extension of this property consists of characterizing a large class of nonlinear systems which are observable independent of the input (called uniformly observable systems). This problem has been studied by [2] in the single output case and visited in [8] by giving a new proof in the case where the nonlinear system is control affine. The authors showed that uniformly observable systems can be transformed into a normal form. Moreover, this normal form has been used in [8] in order to design a high gain observer. The extension of this observer synthesis for non-control affine systems has been stated in the single output case in [11] and in the multi-output case in [21]. In other respects, based on some specific normal forms (having triangular structures), several results on the high gain observer design for MIMO systems exist in the literature: see for instance [10], [14], [19], [20], [28]. In the same spirit, more recently, in [25], [26], [29] and [30] a high gain observer method which allows design of a finite time observer has been proposed. For systems which are not necessarily uniformly observable systems, there is no systematic way permitting to design an observer. Nevertheless, there exist some sufficient conditions based on Lyapunov techniques which permit design of an observer whose gain does not depend on the inputs (see e.g. [1], [13], [17], [23]).

Based on some works mentioned above, in [24], the authors gave sufficient conditions that permit characterization of nonlinear systems which can be converted into higher dimension normal form. The issue is that the proposed class of systems may admit inputs which render these systems unobservable. In order to design an observer for these systems, the authors assumed that the inputs must render the system sufficiently observable in some sense (local regular inputs, see definition 1 below). The gain of the proposed observer was then

The authors are with the Universite de Lyon, F-69003, France; Université Lyon 1; CNRS UMR 5007; Laboratory of Process Control and Chemical Engineering (LAGEP), 43 bd du 11 novembre, 69100 Villeurbanne, France.(e-mail: dufour@lagep.univ-lyon1.fr; flila@lagep.univ-lyon1.fr; hammouri@lagep.univ-lyon1.fr) obtained from a Lyapunov differential equation.

In this paper, we go back to the work of [24] and show that if a system admits a local regular input then the system satisfies the uniform observability structure stated in [21]. Hence a simpler observer may be designed, in the sense that the observer gain does not need a Lyapunov differential equation. Consequently, in this paper, we give a new formulation which permits design of an observer for a class of systems which are not uniformly observable. In this work, the condition that an input must satisfy, in order to guarantee the convergence of the observer, is weaker than the local regular condition given in [24].

This paper is organized as follows: In section 2, we discuss the observer synthesis stated in [24] and give some preliminary results which allow a new formulation that permits design of an observer for a class of non-uniformly observable systems. Finally, in section 3 , we state our main result.

\section{PROBlem STATEMENT AND PRELIMINARY RESULTS}

\section{A. Problem statement}

Consider the following normal form:

$$
\left\{\begin{array}{l}
\dot{z}=F(u, z) \\
y=C z
\end{array}\right.
$$

where the inputs $u($.$) take their values in U \subset \mathbb{R}^{m}$, which is assumed to be compact in the sequel. The state $z($.$) is a column vector of \mathbb{R}^{n}$ which can be decomposed into the form $z=\left(\begin{array}{ccc}z_{1}^{T} \ldots \ldots & \ldots \\ q\end{array}\right)^{T}$ where $z_{i}$ is column vector of $\mathbb{R}^{n_{i}} ; y=C z=z_{1} \in R^{n_{1}} . F(u, z)=$

$$
\begin{aligned}
& \left(\begin{array}{c}
F_{1}(u, z) \\
\vdots \\
F_{q}(u, z)
\end{array}\right) \text {, where: } \\
& \quad F_{i}(u, z)=F_{i}\left(u, z_{1}, \ldots, z_{i+1}\right), \text { for } 1 \leq i \leq q-1
\end{aligned}
$$

which means that $\dot{z}_{i}=F_{i}(u, z)$.

Using the uniform observability concept, the authors in [21] gave a geometric condition (uniform observability structure) that permits the transformation of a nonlinear system into the normal form (1)-(2) with the following additional rank condition:

$$
\operatorname{Rank}\left(\frac{\partial F_{i}}{\partial z_{i+1}}(u, z)\right)=n_{i+1} ; \forall z ; \forall u \in U
$$

Remark 1. From the rank condition (3), we can obviously deduce that $n_{1} \geq \ldots \geq n_{q}$.

The above normal form (1)-(2) together with the rank condition (3) have been used in [21] in order to synthesize an exponential observer. In this paper, we restrict ourselves to the case where $F(u, z)=$ $A(u) z+G(u, z)$. Hence system (1) takes the following particular form:

$$
\left\{\begin{array}{l}
\dot{z}=A(u) z+G(u, z) \\
y=C z=z_{1}
\end{array}\right.
$$

where:

$$
\left\{\begin{array}{c}
C=\left[I_{n_{1}} \ldots 0 \ldots 0\right] \\
A(u)=\left(\begin{array}{cccc}
0 & A_{12}(u) & \ldots & 0 \\
0 & 0 & \ddots & \vdots \\
\vdots & \vdots & \ddots & A_{q-1, q}(u) \\
0 & 0 & \ldots & 0
\end{array}\right) \\
G(u, z)=\left(\begin{array}{c}
G_{1}(u, z) \\
\vdots \\
G_{q}(u, z)
\end{array}\right)
\end{array}\right.
$$


$u \in U, G_{i}(u, z)=G_{i}\left(u, z_{1}, \ldots, z_{i}\right), z_{i} \in \mathbb{R}^{n_{i}}, A_{i, i+1}(u)$ are $n_{i} \times n_{i+1}$ continuous matrices and $I_{n_{1}}$ is the $n_{1} \times n_{1}$ identity matrix.

Remark 2. In order to obtain an extension of the observer design stated in [21], the rank condition given in (3) will be omitted. In particular inequalities $n_{1} \geq \ldots \geq n_{q}$ are not necessarily satisfied, and system (5) may admit inputs which render it unobservable, namely, inputs which do not distinguish between any two different initial states.

\section{Problem formulation:}

As in [24] and many references herein, we will design an observer for systems (5) such that its gain depends only on the pair $(C, A()$.$) ,$ the input $u($.$) and the Lipschitz constant of G$. To do so, consider the set $\mathcal{G}_{c}=\left\{G\right.$, such that $\left\|\frac{\partial G}{\partial z}(u, z)\right\| \leq c, \quad \forall(u, z) \in U \times$ $\left.\mathbb{R}^{n}\right\}$. The observer formulation that we will solve below consists of characterization of a set $\mathcal{U} \subset L^{\infty}\left(\mathbb{R}^{+}, U\right)$ and a system of the form:

$$
\left\{\begin{array}{l}
\dot{\vec{z}}=A(u) \widehat{z}+G(u, \widehat{z})+K(S)(C \widehat{z}-y) \\
\dot{S}=H(u, S)
\end{array}\right.
$$

where $S(t)$ belongs to an open subset of some $\mathbb{R}^{N}$ and $H, K$ are smooth functions, such that system (6) forms an exponential observer for system (5) which converges for every $u \in \mathcal{U}$ and for every $G \in \mathcal{G}_{c}$. This formulation means that the observer gain does not depend on the nonlinear term $G$.

\section{B. Some preliminary results}

In [9] and [24], the authors gave a condition that $u(t)$ must satisfy in order to design an observer which converges independently on the choice of the global Lipschitz term $G$. Such inputs are called local regular inputs and are defined as follows:

Let $\Phi_{u}(t, s)$ be the transition matrix of the state affine system:

$$
\left\{\begin{array}{l}
\dot{z}=A(u) z \\
y=C z
\end{array}\right.
$$

defined by $\frac{d\left(\Phi_{u}(t, s)\right)}{d t}=A(u(t)) \Phi_{u}(t, s)$, with $\Phi_{u}(s, s)=I$ ( $I$ is the identity matrix).

Definition 1. [9], [24] A bounded input $u($.$) is said to be local$ regular input (or locally regular), if there exist $\theta_{0}>0$ and $\alpha>0$, such that for every $\theta \geq \theta_{0}$; for every $t \geq \frac{1}{\theta}$ :

$$
\Psi\left(t-\frac{1}{\theta}, t\right)=\int_{t-\frac{1}{\theta}}^{t} \Phi_{u}^{T}(s, t) C^{T} C \Phi_{u}(s, t) d s \geq \alpha \theta \Delta_{\theta}^{-2}
$$

where $\Psi\left(t-\frac{1}{\theta}, t\right)$ is the Gramian of observability defined on $\left[t-\frac{1}{\theta}, t\right]$, and where:

$$
\Delta_{\theta}=\left(\begin{array}{ccccc}
\theta I_{n_{1}} & 0 & \ldots & & 0 \\
0 & \theta^{2} I_{n_{2}} & \ddots & & \vdots \\
\vdots & \ldots & \ddots & & 0 \\
0 & \ldots & \ldots & \ldots & \theta^{q} I_{n_{q}}
\end{array}\right)
$$

The following result is stated in [24]:

Theorem 1. [24] Assuming that $G$ is a global Lipschitz function and that $u($.$) is locally regular, then an observer for the nonlinear system$ (5) takes the following form:

$$
\left\{\begin{array}{l}
\dot{\widehat{z}}=A(u) \widehat{z}+G(u, \widehat{z})-\Delta_{\theta} S^{-1} C^{T}(C \widehat{z}-y) \\
\dot{S}=-\theta\left(\gamma S+A^{T}(u) S+S A(u)-C^{T} C\right) \\
S(0) \text { is a } n \times n \text { symmetric positive definite (SPD) matrix. }
\end{array}\right.
$$

where $\theta>0$ and $\gamma>0$ are constant parameters.

Remark 3. In section III, we will discuss an example where the particular input $u(t)=\cos (t)$ is not a local regular input, but is a regular input (as defined in definition 2 below).

We end this section by showing (see proposition below) that the local regular input hypothesis (see definition 1) is here a strong one, in the sense that the set of such inputs is an empty one whenever the inequalities $n_{1} \geq \ldots \geq n_{q}$ (which is a consequence of the uniform observability structure stated in (2-(3)) is not satisfied.

Proposition 1. Assuming that there exists an input $u($.$) which is$ locally regular and which is continuous at some $\tau_{0}>0$, then for $2 \leq$ $i \leq q$, we have $\operatorname{Rank}\left(A_{i-1, i}\left(u\left(\tau_{0}\right)\right)\right)=n_{i}$, and since $A_{i-1, i}(u)$ is a $n_{i-1} \times n_{i}$ matrix, it follows that $n_{1} \geq \ldots \geq n_{q}$.

Proof: Since $A(u)$ is a nilpotent matrix $\left(A^{q}(u)=0\right)$ and $C=$ $\left[I_{n_{1}} 0 \ldots 0 \ldots 0\right]$, we deduce that:

$$
C \Phi_{u}(s, t)=\left[E_{1}(t, s) \ldots E_{q}(t, s)\right]
$$

where:

$$
\left\{\begin{aligned}
& E_{1}(t, s)=I_{n_{1}} \\
& E_{2}(t, s)=\int_{t}^{s} A_{12}\left(u\left(t_{1}\right)\right) d t_{1} \\
& \text { and for } 3 \leq i \leq q: \\
& E_{i}(t, s)=\int_{t}^{s} \int_{t}^{t_{1}} \cdots \int_{t}^{t_{i-2}} A_{12}\left(u\left(t_{1}\right)\right) \ldots A_{i-1, i}\left(u\left(t_{i-1}\right)\right) \\
& d t_{1} \ldots d t_{i-1}
\end{aligned}\right.
$$

hence:

$$
\begin{aligned}
& \Psi\left(t-\frac{1}{\theta}, t\right)=\int_{t-\frac{1}{\theta}}^{t} \Phi_{u}^{T}(s, t) C^{T} C \Phi_{u}(s, t) d s \\
& =\left(\begin{array}{cccc}
\psi_{11}\left(t-\frac{1}{\theta}, t\right) & \psi_{12}\left(t-\frac{1}{\theta}, t\right) & \ldots & \psi_{1 q}\left(t-\frac{1}{\theta}, t\right) \\
\psi_{12}^{T}\left(t-\frac{1}{\theta}, t\right) & \psi_{22}\left(t-\frac{1}{\theta}, t\right) & \ldots & \psi_{2 q}\left(t-\frac{1}{\theta}, t\right) \\
\vdots & \vdots & \ddots & \vdots \\
\psi_{1 q}^{T}\left(t-\frac{1}{\theta}, t\right) & \psi_{2 q}^{T}\left(t-\frac{1}{\theta}, t\right) & \ldots & \psi_{q q}\left(t-\frac{1}{\theta}, t\right)
\end{array}\right)
\end{aligned}
$$

where $\psi_{i j}\left(t-\frac{1}{\theta}, t\right)=\int_{t-\frac{1}{\theta}}^{t} E_{i}^{T}(t, s) E_{j}(t, s) d s$.

Condition (8) implies:

$$
\begin{aligned}
& \exists \theta_{0}>0 ; \exists \alpha>0 ; \forall \theta \geq \theta_{0} ; \forall t \geq \frac{1}{\theta} ; \forall i, 1 \leq i \leq q: \\
& \psi_{i i}\left(t-\frac{1}{\theta}, t\right) \geq \frac{\alpha}{\theta^{2 i-1}} I_{n_{i}}
\end{aligned}
$$

$u($.$) is continuous at some \tau_{0}>0$, consider $\theta_{0}$ such that $\tau_{0}>\frac{1}{\theta_{0}}$ and using the fact that $A(u)$ is continuous with respect to $u$, we deduce that for $2 \leq i \leq q$ :

$$
\begin{aligned}
& \left\{\begin{array}{l}
\psi_{i i}\left(\tau_{0}, \theta\right)= \\
\left(\int_{\tau_{0}-\frac{1}{\theta}}^{\tau_{0}} \int_{\tau_{0}}^{s} \ldots \int_{\tau_{0}}^{t_{i-2}} A_{i-1, i}^{T}\left(u\left(t_{i-1}\right)\right) \ldots A_{12}^{T}\left(u\left(t_{1}\right)\right)\right. \\
\left.d t_{1} \ldots d t_{i-1} d s\right) \\
\left(\int_{\tau_{0}-\frac{1}{\theta}}^{\tau_{0}} \int_{\tau_{0}}^{s} \ldots \int_{\tau_{0}}^{t_{i-2}} A_{12}\left(u\left(t_{1}\right)\right) \ldots A_{i-1, i}\left(u\left(t_{i-1}\right)\right)\right. \\
\left.d t_{1} \ldots d t_{i-1} d s\right) \\
=\frac{1}{(2 i-1)((i-1) !)^{2} \theta^{2 i-1}} \\
\left(A_{i-1, i}^{T}\left(u\left(\tau_{0}\right)\right) \ldots A_{12}^{T}\left(u\left(\tau_{0}\right)\right) A_{12}\left(u\left(\tau_{0}\right)\right) \ldots A_{i-1, i}\left(u\left(\tau_{0}\right)\right)\right) \\
+\epsilon_{i}\left(\tau_{0}, \theta\right) \quad \lim _{\theta \rightarrow \infty} \epsilon_{i}\left(\tau_{0}, \theta\right)=0
\end{array}\right. \\
& \text { with: } \quad \text { (16) }
\end{aligned}
$$

Noticing that $A_{i-1, i}^{T}\left(u\left(\tau_{0}\right)\right) \ldots A_{12}^{T}\left(u\left(\tau_{0}\right)\right) A_{12}\left(u\left(\tau_{0}\right)\right) \ldots A_{i-1, i}\left(u\left(\tau_{0}\right)\right)$ together with $\epsilon_{i}\left(\tau_{0}, \theta\right)$ are $n_{i} \times n_{i}$ matrices. Combining (14), (15) and (16), we deduce that: 
$\operatorname{Rank}\left(\left(A_{i-1, i}^{T}\left(u\left(\tau_{0}\right)\right) \ldots A_{12}^{T}\left(u\left(\tau_{0}\right)\right)\right)\left(A_{12}\left(u\left(\tau_{0}\right)\right) \ldots A_{i-1, i}\left(u\left(\tau_{0}\right)\right)\right)\right)$ $=n_{i}$.

Finally $\operatorname{Rank}\left(A_{i-1, i}().\right) \leq \min \left\{n_{i}, n_{i-1}\right\}$ yields to $n_{1} \geq \ldots \geq n_{q}$.

\section{OBSERVER SYNTHESIS BASED ON REGULAR INPUTS}

In proposition 1 above, we have shown that the local regular input assumption which is used in theorem 1) implies that $n_{1} \geq \ldots \geq n_{q}$, which restricts the class of systems (5). In this section, this local regular assumption is replaced by a weaker one, and an observer for the class of systems (5) is proposed. To do so, let us consider the differential equation defined on the manifold $\mathcal{S}^{+}$of SPD matrices:

$$
\left\{\begin{array}{l}
\dot{S}=-\theta S-A^{T}(u) S-S A(u)+C^{T} C \\
S(0) \in \mathcal{S}^{+}
\end{array}\right.
$$

where $A(u)$ is the $n \times n$ matrix given in (5). A simple calculation gives:

$$
\begin{aligned}
S(t)= & e^{-\theta t} \Phi_{u}^{T}(0, t) S(0) \Phi_{u}(0, t) \\
& +\int_{0}^{t} e^{-\theta(t-s)} \Phi_{u}^{T}(s, t) C^{T} C \Phi_{u}(s, t) d s
\end{aligned}
$$

Since, $\Phi_{u}^{T}(0, t) S(0) \Phi_{u}(0, t)$ is a SPD matrix and that the integral term of (18) is a symmetric positive matrix, it follows that $S(t)$ is a SPD matrix. In the sequel, we will consider the following matrix:

$$
\begin{gathered}
\mathcal{P}(t)=\int_{0}^{t} e^{-\theta(t-s)} \Phi_{u}^{T}(s, t) C^{T} C \Phi_{u}(s, t) d s= \\
\left(\begin{array}{cccc}
\mathcal{P}_{11}(t) & \mathcal{P}_{12}(t) & \ldots & \mathcal{P}_{1 n}(t) \\
\mathcal{P}_{12}^{T}(t) & \mathcal{P}_{22}(t) & \ldots & \mathcal{P}_{2 n}(t) \\
\vdots & \vdots & \ddots & \vdots \\
\mathcal{P}_{1 n}^{T}(t) & \mathcal{P}_{2 n}^{T}(t) & \ldots & \mathcal{P}_{n n}(t)
\end{array}\right)
\end{gathered}
$$

Remark 4. Let $U$ a bounded subset of $\mathbb{R}^{m}$ in which $u($.$) takes its$ values and $\theta>0$, then:

a) There exists a constant $\kappa>0$, s.t. for every input $u$ which takes its values in $U$, we have:

$$
\mathcal{P}(t) \leq \kappa I
$$

b) $S(t)$ and $\mathcal{P}(t)$ have the same behavior for large $t$. More precisely, there exists a constant $\omega>0$ which only depends on $U$ such that:

$$
S(t)-\omega e^{-\frac{\theta t}{2}} I \leq \mathcal{P}(t) \leq S(t)+\omega e^{-\frac{\theta t}{2}} I
$$

Proof: The inequality (20) comes from the boundedness of $A(u()$.$) and the expressions (11)-(12). The inequalities in (21) are$ direct consequence of expression (18).

In the sequel, $\Gamma(t)$ denotes the $n \times n$ symmetric positive block diagonal matrix:

$$
\Gamma(t)=\left(\begin{array}{ccc}
\mathcal{P}_{11}(t) & \ldots & 0 \\
\vdots & \ddots & \vdots \\
0 & \ldots & \mathcal{P}_{q q}(t)
\end{array}\right)
$$

The $\mathcal{P}_{i i}$ are given by:

- $\mathcal{P}_{11}(t)=\left(\int_{0}^{t} e^{-\theta(t-s)} d s\right) I_{n_{1}}$

- For $i \geq 2: \mathcal{P}_{i i}(t)=\ldots$

$$
\begin{aligned}
& \int_{0}^{t} e^{-\theta(t-s)}[ \\
& \left(\int_{s}^{t} \int_{s}^{t_{1}} \ldots \int_{s}^{t_{i-2}} A_{i-1, i}^{T}\left(u\left(t_{i-1}\right)\right) \ldots A_{12}^{T}\left(u\left(t_{1}\right)\right) d t_{1} \ldots d t_{i-1}\right) \\
& \left(\int_{s}^{t} \int_{s}^{t_{1}} \ldots \int_{s}^{t_{i-2}} A_{12}\left(u\left(t_{1}\right)\right) \ldots A_{i-1, i}\left(u\left(t_{i-1}\right)\right) d t_{1} \ldots d t_{i-1}\right) \\
& ] d s
\end{aligned}
$$

Definition 2. A bounded input $u($.$) is said to be a regular input if,$ and only if, $\exists \theta_{0}>0 ; \exists T_{0}>0 ; \exists t_{0} \geq T_{0} ; \exists \alpha_{0} ; \forall \theta>\theta_{0} ; \forall t \geq t_{0}$, we have:

i) $\int_{t-T_{0}}^{t} \Phi_{u}^{T}(s, t) C^{T} C \Phi_{u}(s, t) d s \geq \alpha_{0} I$, where $I$ is the identity matrix.

ii) $\Gamma(t) \leq \alpha(\theta) \mathcal{P}(t)$, for some $\alpha(\theta)$ such that $\lim _{\theta \rightarrow \infty} \frac{\alpha(\theta)}{\theta^{2}}=0$.

iii) $\exists \gamma>0,\left\|\mathcal{P}_{j j}^{-1}(t)\right\|\left\|\mathcal{P}_{i i}(t)\right\| \leq \gamma$, for $1 \leq j \leq i$.

Lemma 1. Consider $t_{0}$ of definition 2, there exist two constants $\eta_{1}>0, \eta_{2}>0$, such that for every $t \geq t_{0}$, we have:

$$
\mathcal{P}(t) \geq \eta_{2} \int_{t-T_{0}}^{t} e^{-\theta(t-s)} \Phi_{u}^{T}(s, t) C^{T} C \Phi_{u}(s, t) d s \geq \eta_{3} I
$$

where $\eta_{3}=\alpha_{0} \eta_{2} e^{-\theta T_{0}}$, and:

$$
\eta_{1} \mathcal{P}(t) \leq S(t) \leq \eta_{2} \mathcal{P}(t)
$$

Proof: (23) comes from the definition of $\mathcal{P}$ and i) of the above definition. (24) is deduced from the definition of $S(t), \mathcal{P}(t)$ and (23).

Remark 5. The set of regular inputs contains the set of local regular inputs.

Proof: Let us show that if $u$ is a local regular input, then i), ii) and iii) of definition 2 are satisfied.

i) Let $u$ be a local regular input, from (8), we have: $\Psi(t-$ $\left.\frac{1}{\theta}, t\right)=\int_{t-\frac{1}{\theta}}^{t} \Phi_{u}^{T}(s, t) C^{T} C \Phi_{u}(s, t) d s \geq \alpha \theta \Delta_{\theta}^{-2}$. Hence i) of definition 2 is satisfied for $T_{0}=\theta^{-1}$.

ii) On the one hand, using the expression of $\mathcal{P}_{i i}(t)$ and the fact that the $A_{k l}(u(t))$ are bounded, we can show that $\mathcal{P}_{i i}(t) \leq$ $\frac{a}{\theta^{2 i-1}} I_{n_{i}}$, for some constant $a$ which does not depend on $\theta$. Hence, $\Gamma(t) \leq a \theta \Delta_{\theta}^{-2}$. On the other hand, since $u$ is local regular, we have $\mathcal{P}(t) \geq e^{-1} \Psi\left(t-\frac{1}{\theta}, t\right) \geq \alpha e^{-1} \theta \Delta_{\theta}^{-2}$ $\left(\mathcal{P}_{i i}(t) \geq \frac{\alpha e^{-1}}{\theta^{2 i-1}} I_{n_{i}}\right)$. Combining these two facts, we deduce $\Gamma(t) \leq \frac{a e}{\alpha} \mathcal{P}(t)$, which is exactly condition ii) of definition 2.

iii) From above we know that $\frac{\widetilde{a}}{\theta^{2 i-1}} I_{n_{i}} \leq \mathcal{P}_{i i}(t) \leq \frac{a}{\theta^{2 i-1}} I_{n_{i}}$ for some constants $a$ and $\widetilde{a}$. Thus iii) of definition 2 is fulfilled.

Example 1. For the following example, we show that $u(t)=\cos (t)$ is not a local regular input, but is a regular input.

$$
\left\{\begin{array}{l}
\dot{x}=\left(\begin{array}{cc}
0 & u \\
0 & 0
\end{array}\right) x \\
y=\left(\begin{array}{ll}
1 & 0
\end{array}\right) x
\end{array}\right.
$$

The fact that $\cos (t)$ is not a local regular input can be obtained as follows:

The transition matrix of (25) is $\left(\begin{array}{cc}1 & \int_{s}^{t} \cos (\tau) d \tau \\ 0 & 1\end{array}\right)$ and the term $\Psi_{22}(t)$ of the Gramian given in (8) is $\int_{t-\frac{1}{\theta}}^{t}\left(\int_{s}^{t} \cos (\tau) d \tau\right)^{2} d s$. At $t=k \pi+\frac{\pi}{2}$, we can verify that $\Psi_{22}(t)=\int_{0}^{\frac{1}{\theta}} \xi^{4} O(\xi) d \xi$, for some bounded function $O($.$) . Hence, for \theta$ sufficiently large, $\Psi_{22}(t) \leq \frac{c}{\theta^{5}}$, where $c>0$ is a constant which does not depend on $\theta$. Thus condition (8) cannot be satisfied. Consequently, $\cos (t)$ is not a regular input for (25).

In the appendix, we show that $u(t)=\cos (t)$ is a regular input for this system.

Our candidate observer for system (5) takes the following form:

$$
\left\{\begin{array}{l}
\dot{\widehat{z}}=A(u) \widehat{z}+G(u, \widehat{z})-S^{-1} C^{T}(C \widehat{z}-y) \\
\dot{S}=-\theta S-A^{T}(u) S-S A(u)+C^{T} C \\
S(0) \text { is a SPD matrix }
\end{array}\right.
$$


As above, the control set $U$ is bounded and $\mathcal{G}_{c}=$ $\left\{G\right.$, such that $\left.\left\|\frac{\partial G}{\partial z}(u, z)\right\| \leq c, \quad \forall(u, z) \in U \times \mathbb{R}^{n}\right\}$, for some fixed constant $c>0$.

Now we can state our main result:

Theorem 2. Let $G \in \mathcal{G}_{c}$ and let $u($.$) be a regular input. Then there$ exists $\theta_{0}>0$ s.t. for every $\theta \geq \theta_{0}$; there exists two constants $\mu_{1}(\theta)>$ $0, \mu_{2}(\theta)>0$ such that $\|\widehat{z}(t)-z(t)\| \leq \mu_{1}(\theta) e^{-\mu_{2}(\theta) t}\|\widehat{z}(0)-z(0)\|$, where $z(t)$ and $\widehat{z}(t)$ are the respective trajectories of systems (5) and (26).

Proof: Setting $e(t)=\widehat{z}(t)-z(t)$, from (5)-(26), we get:

$$
\left\{\begin{array}{l}
\dot{e}=\left(A(u)-S^{-1} C^{T} C\right) e+\delta(G) \\
\dot{S}=-\theta S-A^{T}(u) S-S A(u)+C^{T} C
\end{array}\right.
$$

where $\delta(G)=G(u, \widehat{z})-G(u, z)$. From (18), we know that $S(t)$ is a SPD matrix. Using the Cholesky decomposition we can set: $S(t)=\Lambda(t) \Lambda^{T}(t)$, where $\Lambda(t)$ is a lower triangular matrix, moreover its diagonal terms are strictly positive. Now setting $\epsilon(t)=\Lambda^{T}(t) e(t)$, we get:

$$
\dot{\epsilon}=\left(\Lambda^{T} A(u) \Lambda^{-T}-\Lambda^{-1} C^{T} C \Lambda^{-T}\right) \epsilon+\Lambda^{T} \delta(G)+\dot{\Lambda}^{T} \Lambda^{-T} \epsilon
$$

where $\Lambda^{-T}$ denotes the inverse of $\Lambda^{T}$. Using again $S(t)=$ $\Lambda(t) \Lambda^{T}(t)$ and the fact that $S(t)$ is a solution of the second equation of (26), we obtain:

$$
\dot{\Lambda} \Lambda^{T}+\Lambda \dot{\Lambda}^{T}=-\theta \Lambda \Lambda^{T}-A^{T}(u) \Lambda \Lambda^{T}-\Lambda \Lambda^{T} A(u)+C^{T} C
$$

Let us multiply both sides of (29): to the left by $\Lambda^{-1}$, and by $\Lambda^{-T}$ to the right, we get:

$$
\begin{aligned}
& \Lambda^{-1} \dot{\Lambda}+\dot{\Lambda}^{T} \Lambda^{-T}=-\theta I-\Lambda^{-1} A^{T}(u) \Lambda-\Lambda^{T} A(u) \Lambda^{-T}+ \\
& \Lambda^{-1} C^{T} C \Lambda^{-T}
\end{aligned}
$$

Now setting $V(t)=\epsilon^{T}(t) \epsilon(t)$ :

$$
\left\{\begin{aligned}
\dot{V} & =\dot{\epsilon}^{T} \epsilon+\epsilon^{T} \dot{\epsilon} \\
& =\epsilon^{T}\left[\Lambda^{-1} A^{T}(u) \Lambda-\Lambda^{-1} C^{T} C \Lambda^{-T}\right] \epsilon \\
& +\epsilon^{T}\left[\Lambda^{T} A(u) \Lambda^{-T}-\Lambda^{-1} C^{T} C \Lambda^{-T}\right] \epsilon \\
& +2 \epsilon^{T} \Lambda^{T} \delta(G) \\
& +\epsilon^{T}\left[\Lambda^{-1} \dot{\Lambda}+\dot{\Lambda}^{T} \Lambda^{-T}\right] \epsilon
\end{aligned}\right.
$$

Combining (30) and (31), it follows:

$$
\left\{\begin{array}{l}
\dot{V}=-\theta V+2 \epsilon^{T} \Lambda^{T} \delta(G)-\epsilon^{T} \Lambda^{-1} C^{T} C \Lambda^{-T} \epsilon \\
\leq-\theta V+2 \sqrt{V}\left\|\Lambda^{T} \delta(G)\right\|
\end{array}\right.
$$

where $\|$.$\| denotes the Euclidean norm.$

Combining condition i) of definition 2 with the second inequality of (24), we obtain:

$$
\forall t \geq t_{0}, S(t)=\Lambda(t) \Lambda^{T}(t) \leq \eta_{2} \mathcal{P}(t)
$$

Thus (32)-(33), yield to:

$$
\dot{V} \leq-\theta V+2 \sqrt{a} \sqrt{V} \sqrt{(\delta(G))^{T} \mathcal{P}(t) \delta(G)}, \forall t \geq t_{0}
$$

Since $\mathcal{P}(t)$ is a SPD matrix for $t \geq t_{0}$ (condition i) of definition 2), we deduce that $2 z_{i}^{T} \mathcal{P}_{i j}(t) z_{j} \leq z_{i}^{T} \mathcal{P}_{i i}(t) z_{i}+$ $z_{j}^{T} \mathcal{P}_{j j}(t) z_{j}$. Thus $\mathcal{P}(t) \leq a(q) \Gamma(t)$, where $\Gamma(t)$ is the diagonal block matrix of $\mathcal{P}(t)$ (defined in (22)) and $a(q)$ is a positive constant which depends only on $q$. Hence $(\delta(G))^{T} \mathcal{P}(t) \delta(G) \leq$ $a(q) \sum_{i=1}^{q}(\delta(G))_{i}^{T} \mathcal{P}_{i i}(t) \delta(G)_{i}$. Now using the mean value theorem, we obtain: $\delta(G)_{i}=\sum_{j=1}^{i} M_{i j}(.) e_{j}$, where $e_{j}=\widehat{z}_{j}-z_{j}$ and $M_{i j}($. is a $n_{i} \times n_{j}$ matrix depending only on $(u, z, \widehat{z})$ and which is bounded (since $G \in \mathcal{G}_{c}$ ). Hence the following hold:

$$
\left\{\begin{array}{l}
(\delta(G))_{i}^{T} \mathcal{P}_{i i}(t) \delta(G)_{i}=\sum_{l, k=1}^{i} e_{k}^{T} M_{i k}^{T}(.) \mathcal{P}_{i i}(t) M_{i l}(.) e_{l} \\
\leq M\left\|\mathcal{P}_{i i}(t)\right\| \sum_{l=1}^{i} e_{l}^{T} e_{l}, \mathrm{M} \text { is a constant }
\end{array}\right.
$$

Since the $\mathcal{P}_{i i}$ 's are $n_{i} \times n_{i}$ SPD matrices, using the Cholesky decomposition, we get $\mathcal{P}_{i i}(t)=L_{i} L_{i}^{T}$ where $L_{i}$ is a lower triangular matrix. Set $\widetilde{\epsilon}_{i}=L_{i} e_{i}$ and $\widetilde{\epsilon}=\left(\widetilde{\epsilon}_{1}^{T} \ldots \widetilde{\epsilon}_{q}^{T}\right)^{T}$, from (35) and inequality iii) of definition 2 , it follows that for every $t \geq t_{0}$ :

$\left\{\begin{array}{l}(\delta(G))_{i}^{T} \mathcal{P}_{i i}(t) \delta(G)_{i} \leq M\left\|\mathcal{P}_{i i}(t)\right\| \sum_{l=1}^{i}\left\|\mathcal{P}_{l l}^{-1}(t)\right\| \widetilde{\epsilon}_{l}^{T} \widetilde{\epsilon}_{l} \\ \leq b \sum_{l=1}^{i} \widetilde{\epsilon}_{l}^{T} \widetilde{\epsilon}_{l}, \text { where } b>0 \text { is a constant which not depend on } \theta\end{array}\right.$

Combining (34) and (36), there exists a constant $\rho>0$, which does not depend on $\theta$, such that:

$$
\forall t \geq t_{0}, \quad \dot{V} \leq-\theta V+\rho \sqrt{V} \sqrt{\widetilde{\epsilon}^{T} \widetilde{\epsilon}}
$$

From ii) of definition 2, the following holds for $\theta \geq \theta_{0}$ and $t \geq t_{0}$ :

$$
\Gamma(t) \leq \alpha(\theta) \mathcal{P}(t), \text { with } \lim _{\theta \rightarrow \infty} \frac{\alpha(\theta)}{\theta^{2}}=0
$$

On one hand, using the definition of $\widetilde{\epsilon}$ and $\Gamma(t)$, we get:

$$
\widetilde{\epsilon}^{T} \widetilde{\epsilon}=e^{T} \Gamma(t) e \leq \alpha(\theta) e^{T} \mathcal{P} e
$$

On the other hand, using the first inequality of (24), we get:

$e^{T}(t) \mathcal{P}(t) e(t) \leq \eta_{1}^{-1} e^{T}(t) S(t) e(t)=\eta_{1}^{-1} V(t)$, for every $t \geq t_{0}$

Now combining (37), (39) and (40), we obtain:

$$
\dot{V} \leq-\theta V+\rho \sqrt{\frac{\alpha(\theta)}{\eta_{1}}} V
$$

But $\lim _{\theta \rightarrow \infty} \frac{\alpha(\theta)}{\theta^{2}}=0$, hence for $\theta_{0}$ sufficiently large and $\theta \geq \theta_{0}$, we deduce that $V(t) \leq \widetilde{\mu}_{1} e^{-\widetilde{\mu}_{2} t} V(0)$, for some constants $\widetilde{\mu}_{1}>0$, $\widetilde{\mu}_{2}>0$ depending on $\theta$. Finally, using the first inequality of (24) and inequality (23), it follows that $\|e(t)\| \leq \mu_{1}(\theta) e^{-\mu_{2}(\theta) t}\|e(0)\|$.

\section{CONCLUSION}

In this paper, an observer design for systems that admit inputs which render them unobservable has been investigated. It has been showed that if the input satisfy some observability assumption (regular input), one may design an observer where the gain requires the resolution of a Lyapunov differential equation which does not depend on the nonlinear term.

\section{APPENDIX}

In the example 1 in section III, we have seen that $u(t)=\cos (t)$ is not a local regular input for the considered model of this example. Here, for the same model, we show that $u(t)=\cos (t)$ is a regular input.

$$
\left\{\begin{array}{l}
\dot{x}=\left(\begin{array}{ll}
0 & u \\
0 & 0
\end{array}\right) x \\
y=\left(\begin{array}{ll}
1 & 0
\end{array}\right) x
\end{array}\right.
$$

Let us check conditions i), ii) and iii) of definition 2 :

1) Condition i): The transition matrix is $\Phi(s, t)=$ $\left(\begin{array}{cc}1 & \int_{s}^{t} \cos (\tau) d \tau \\ 0 & 1\end{array}\right)$

Set $T_{0}=2 \pi$, the Gramian of observability on the interval $[t-2 \pi, t]$ is given by $G(u, t-2 \pi, t)=$ $\left(\begin{array}{cc}2 \pi & \int_{t-2 \pi}^{t}\left(\int_{s}^{t} \cos (\tau) d \tau\right) d s \\ \int_{t-2 \pi}^{t}\left(\int_{s}^{t} \cos (\tau) d \tau\right) d s & \int_{t-2 \pi}^{t}\left(\int_{s}^{t} \cos (\tau) d \tau\right)^{2} d s\end{array}\right)=$ 
$\pi\left(\begin{array}{cc}2 & -2 \sin (t) \\ -2 \sin (t) & 1+2 \sin ^{2}(t)\end{array}\right), \quad$ and $\quad$ clearly, $G(u, t-2 \pi, t) \geq \alpha_{0} I$, for some constant $\alpha_{0}>0$. This ends the proof of condition i) of definition 2 .

2) Condition ii): The matrix $\mathcal{P}(t)$ defined in (19) is given by: $\mathcal{P}(t)=$ $\left(\begin{array}{cc}\int_{0}^{t} e^{-\theta(t-s)} d s & \int_{0}^{t} e^{-\theta(t-s)}\left(\int_{s}^{t} \cos (\tau) d \tau\right) d s \\ \int_{0}^{t} e^{-\theta(t-s)}\left(\int_{s}^{t} \cos (\tau) d \tau\right) d s & \int_{0}^{t} e^{-\theta(t-s)}\left(\int_{s}^{t} \cos (\tau) d \tau\right)^{2} d s\end{array}\right)$. Hence we can obtain the decomposition $\mathcal{P}(t)=\widetilde{\mathcal{P}}(t)+M(t)$, where $\widetilde{\mathcal{P}}(t)$ is:

$$
\left(\begin{array}{cc}
\frac{1}{\theta} & \frac{\theta \cos (t)+\sin (t)}{\theta\left(\theta^{2}+1\right)} \\
\frac{\theta \cos (t)+\sin (t)}{\theta\left(\theta^{2}+1\right)} & \widetilde{\mathcal{P}}_{22}(t)
\end{array}\right)
$$

where $\widetilde{\mathcal{P}}_{22}(t)=\frac{2 \theta^{2} \cos ^{2}(t)+4 \sin ^{2}(t)+6 \theta \sin (t) \cos (t)+2}{\theta\left(1+\theta^{2}\right)\left(4+\theta^{2}\right)}$ and $\|M(t)\| \leq \tilde{a} e^{-\frac{\theta}{2} t}$, for some constant $\tilde{a}$ which does not depend on $\theta$. Consequently, it suffices to verify condition ii) of definition 2 for $\widetilde{\mathcal{P}}(t)$ and its block diagonal matrix $\widetilde{\Gamma}(t)$ :

$\widetilde{\Gamma}(t)=\left(\begin{array}{cc}\frac{1}{\theta} & 0 \\ 0 & \frac{2 \theta^{2} \cos ^{2}(t)+4 \sin ^{2}(t)+6 \theta \sin (t) \cos (t)+2}{\theta\left(1+\theta^{2}\right)\left(4+\theta^{2}\right)}\end{array}\right.$

Since $\widetilde{\Gamma}(t)$ is invertible, to show that $\widetilde{\Gamma}(t) \leq \alpha(\theta) \widetilde{\mathcal{P}}(t)$ (for some constant $\alpha(\theta)$ s.t. $\lim _{\theta \rightarrow \infty} \frac{\alpha(\theta)}{\theta^{2}}=0$ ), it suffices to show the following:

$$
P_{\theta}(t)=\widetilde{\Gamma}^{-\frac{1}{2}}(t) \widetilde{\mathcal{P}}(t) \widetilde{\Gamma}^{-\frac{1}{2}}(t) \geq \alpha^{-1}(\theta) I
$$

Consider the following elementary relation:

$$
z^{T} P_{\theta}(t) z=z_{1}^{2}+z_{2}^{2}+2 a(\theta, t) z_{1} z_{2} \geq(1-|a(\theta, t)|)\|z\|^{2}
$$

where:

$$
a(\theta, t)=\frac{(\theta \cos (t)+\sin (t)) \sqrt{4+\theta^{2}}}{\sqrt{1+\theta^{2}} \sqrt{2} \sqrt{\left(\theta \cos (t)+\frac{3}{2} \sin (t)\right)^{2}+1-\frac{1}{4} \sin ^{2}(t)}}
$$

or,

$$
\left\{\begin{array}{l}
\frac{\left(1+\theta^{2}\right)}{\left(4+\theta^{2}\right)} a^{2}(\theta, t)= \\
\frac{(\theta \cos (t)+\sin (t))^{2}}{2\left(\left(\theta \cos (t)+\frac{3}{2} \sin (t)\right)^{2}+1-\frac{1}{4} \sin ^{2}(t)\right)}= \\
\frac{(\theta \cos (t)+\sin (t))^{2}}{2\left[(\theta \cos (t)+\sin (t))^{2}+2(\theta \cos (t)+\sin (t)) \sin (t)+1\right]}
\end{array}\right.
$$

In order to check inequality (45), it suffices to show that:

$$
\left.\exists \alpha_{0} \in\right] 0,1\left[; \exists \theta_{0}>0 ; \forall t \geq 0, a^{2}(\theta, t) \leq \alpha_{0}\right.
$$

If $\theta \cos (t)+\sin (t)=0$, then $a^{2}(\theta, t)=0$ and hence (49) is satisfied. Now assuming that $\theta \cos (t)+\sin (t) \neq 0$, setting $Z=(\theta \cos (t)+\sin (t))^{-1}$ and $s=\sin (t)$, we obtain:

$a^{2}(\theta, t)=\frac{\left(4+\theta^{2}\right)}{\left(1+\theta^{2}\right)} \frac{1}{2\left(1+s Z+Z^{2}\right)}$. Since $\lim _{\theta \rightarrow \infty} \frac{\left(4+\theta^{2}\right)}{\left(1+\theta^{2}\right)}=$ 1 , in order to achieve (49), it suffices to show that $2(1+s Z+$ $\left.Z^{2}\right) \geq \frac{3}{2}$. This last inequality comes from the fact that $2 Z^{2}+$ $2 s Z+\frac{1}{2} \geq 0$, since $|s| \leq 1$. This ends the proof of condition ii) of definition 2 .
3) Condition iii): Using again the decomposition $\mathcal{P}(t)=\widetilde{\mathcal{P}}(t)+$ $M(t)$ given in the above step 2), it suffices to check condition iii) of definition 2 for the diagonal elements of $\widetilde{\mathcal{P}}$ :

$$
\left\{\begin{array}{l}
\widetilde{\mathcal{P}}_{11}=\frac{1}{\theta} \\
\widetilde{\mathcal{P}}_{22}=\frac{4 \theta^{2} \cos ^{2}(t)+4\left(1+2 \sin ^{2}(t)\right)+10 \theta \sin (t) \cos (t)}{2 \theta\left(1+\theta^{2}\right)\left(4+\theta^{2}\right)}
\end{array}\right.
$$

Clearly, it is not difficult to verify that $\widetilde{\mathcal{P}}_{11}^{-1} \widetilde{\mathcal{P}}_{22} \leq \gamma$, where $\gamma$ is a positive constant which does not depend on $\theta$ (since $|s| \leq 1)$. This ends the proof of condition iii) of definition 2 .

\section{REFERENCES}

[1] F.E. Thau, "Observing the state of nonlinear dynamics systems", International Journal of Control, vol. 17, pp. 471-479. 1973.

[2] J.P. Gauthier and G. Bornard, "Observability for any $\mathrm{u}(\mathrm{t})$ of a class of nonlinear systems", IEEE Transactions on Automatic Control, vol. 26, pp. 922-926, 1981.

[3] A.J. Krener and A. Isidori, "Linearization by output injection and nonlinear observers", Systems and Control Letters, vol. 3, pp. 47-52, 1983.

[4] A.J. Krener and W. Respondek, "Nonlinear observer with linearizable error dynamics", SIAM Journal of Control and Optimization, vol. 30, pp. 197-216, 1985.

[5] H. Hammouri and J-P. Gauthier, "Bilinearization up to output injection", Systems and Control Letters, vol. 11, no. 2, pp. 139-149, 1988.

[6] X.H. Xia and W.B. Gao, "Nonlinear observer with linearizable error dynamics", SIAM Journal on Control and Optimization, vol. 27, pp. 199216, 1989.

[7] J. P. Gauthier and H. Hammouri, "Global time-varying linearization up to output injection", SIAM Journal of Control \& Optimization., vol. 30, no. 6, pp. 1295-1310, 1992.

[8] J. P. Gauthier and H. Hammouri and S. Othman, "A simple observer for nonlinear systems - Application to bioreactors", IEEE Transactions on Automatic Control, vol. 37, no. 6, pp. 875-880, 1992.

[9] G. Bornard, F. Celle-Couenne and G. Gilles, "Observabilité et observateurs", in Systèmes Non Linéaires, A. J. Fossard, D. Normand-Cyrot, eds, Modélisation - Estimation, Masson, 1993, 1, 177-221.

[10] G. Ciccarella, M. Dalla Mora and A. Germani, "A Luenberger-like observer for nonlinear systems", Systems and Control Letters, 1993, vol. 47, no. 3, pp. 537-556, 1993.

[11] J. P. Gauthier and I. Kupka, "Observability and observers for nonlinear systems", SIAM Journal of Control \& Optimization, vol. 32, pp. 975-997, 1994.

[12] H. Hammouri and M. Kinnaert, "Observability for systems with more outputs than inputs", Systems and Control Letters, vol. 28, pp. 151-157, 1996.

[13] G. Besançon and H. Hammouri, "On uniform observation of nonuniformly observable systems", Systems Control Letters, vol. 29, pp. 9-19, 1996.

[14] J.P. Gauthier and I. Kupka, "Observability for systems with more outputs than inputs", Mathematische Zeitschrift, vol. 223, pp. 47-78, 1996.

[15] F. Plestan and A. Glumineau, "Linearization by generalized input output injection", Systems and Control Letters, vol. 31, pp. 115-128, 1997.

[16] G. Besançon and H. Hammouri, "On observer design for interconnected systems", Journal of Mathematical Systems, Estimation and Control, vol. 8, no. 4, 1998.

[17] R. Rajamani, "Observers for Lipschitz nonlinear systems", IEEE Transactions on Automatic Control, vol. 43, no. 3, pp. 397-401, 1998.

[18] G. Besançon, H. Hammouri and S. Benamor, "State equivalence of discrete-time nonlinear control systems to state affine form up to output injection", Systems and Control Letters, vol. 33, no 1, pp. 1-10, 1998.

[19] H. Shim, I.S. Young and Jin H. Seo, "Semi-global observer for multioutput nonlinear systems", Systems and Control Letters, vol. 42, pp. 233$244,2001$.

[20] H. Hammouri, B. Targui and F. Armanet, "High gain observer based on a triangular structure", International Journal of Robust and Nonlinear Control, vol. 12, no. 6, 497-518, 2002.

[21] H. Hammouri and M. Farza, "Nonlinear observers for locally uniformly observable systems", ESAIM: Control, Optimization and Calculus of Variations, vol. 9, pp. 353-370, 2003.

[22] W. Respondek, A. Pogromsky and H. Nijmeijer, "Time scaling for observer design with linearization error dynamics", IEEE Transactions on Automatic Control, vol. 3, pp. 199-216, 2004. 
[23] A. Alessandri, "Design of observers for Lipschitz nonlinear systems using LMI" in SNOLCOS, IFAC Symposium on Nonlinear Control Systems, 2004.

[24] G. Besançon and A. Ticlea, "An immersion-based observer design for rank-observable nonlinear systems", IEEE Transactions on Automatic Control, vol. 52, no. 1, pp. 83-88, 2007.

[25] Y. Shen and X. Xia, "Semi-global finite-time observers for nonlinear systems", Automatica, vol. 44, pp. 3152- 3156, 2008.

[26] Y. Shen and Y. Huang, "Uniformly observable and globally Lipschitzian nonlinear systems admit global finite time observers", IEEE Transactions on Automatic Control, vol. 54, pp. 2621-2625, 2009.

[27] D. Boutat, A. Benali, H. Hammouri and K. Busawon, "New algorithm for observer error linearization with a diffeomorphism on the outputs", Automatica, vol. 45, no. 10, pp. 2187-2193, 2009.

[28] H. Hammouri, G. Bornard and K. Busawon, "High gain observer for structured multi-output nonlinear systems", IEEE Transactions on Automatic Control, vol. 55, no. 4, pp. 987-992, 2010.

[29] T. Menard, E. Moulay and W. Perruquetti, "A global finite-time observers for nonlinear systems" IEEE Transactions on Automatic Control, vol. 55, pp. 1500-1506, 2010.

[30] Y. Shen, W. Shen, M. Jiang and Y. Huang. "Semi-global finite-time observers for multi-output nonlinear systems", International Journal of Robust and Nonlinear Control, vol. 20, no. 7, pp. 789-801, 2010. 